\title{
Limits on the Masses of Supermassive Black Holes in 105 Nearby Galaxies
}

\author{
E. M. Corsini ${ }^{1}$, A. Beifiori ${ }^{1}$, M. Sarzi ${ }^{2}$, E. Dalla Bontà ${ }^{1}$, A. Pizzella ${ }^{1}$, \\ L. Coccato ${ }^{3}$, and F. Bertola ${ }^{1}$ \\ ${ }^{1}$ Dipartimento di Astronomia, Università di Padova, Vicolo dell'Osservatorio 3, I-35122 \\ Padova, Italy \\ Email: enricomaria.corsini@unipd.it \\ ${ }^{2}$ Centre for Astrophysics Research, University of Hertfordshire, College Lane, Hatfield AL10 \\ $9 \mathrm{AB}, \mathrm{UK}$ \\ ${ }^{3}$ Max-Planck-Institut für extraterrestrische Physik, Giessenbachstrasse 1, D-85748 Garching \\ bei München, Germany
}

Keywords. black hole physics, galaxies: kinematics and dynamics, galaxies: nuclei

Secure measurements of the mass of the central supermassive black hole, $M_{\mathrm{BH}}$, in external galaxies are traditionally obtained through the modeling of the stellar and/or gaseous kinematics, most often derived using Hubble Space Telescope (HST) observations in the optical domain. The modeling of the nuclear ionized-gas kinematics has led to accurate $M_{\mathrm{BH}}$ measurements at a relatively cheap cost in terms of observation time compared to stellar-dynamical $M_{\mathrm{BH}}$ determinations. But only a handful of the objects have turned out to have sufficiently regular gas velocity fields for the purpose of modeling. Nevertheless, the HST archive contains a yet untapped resource that can be used to better constrain the $M_{\mathrm{BH}}$ budget across the different morphological types of galaxies, which consists of the vast number of the Space Telescope Imaging Spectrograph (STIS) spectra from which a central emission-line width can be measured. These data allow to put an upper limit on $M_{\mathrm{BH}}$ for a large number of galaxies and promise to compensate for the lack of exact measurements when studying the $M_{\mathrm{BH}}$-host galaxy relationships.

For this reason, we used STIS to obtain $\mathrm{H} \alpha$ spectra of the nuclei of 105 nearby $(D<100 \mathrm{Mpc})$ galaxies spanning a wide range of Hubble types $(\mathrm{E}-\mathrm{Sc})$ and values of the central stellar velocity dispersion, $\sigma_{\mathrm{c}}\left(58-419 \mathrm{~km} \mathrm{~s}^{-1}\right)$. We obtained stringent upper bounds on their black hole masses (Beifiori et al. 2009). For the vast majority of the objects, the derived upper limits on $M_{\mathrm{BH}}$ run parallel to and above the well-known $M_{\mathrm{BH}}-\sigma_{\mathrm{c}}$ relation, independent of the galaxy distance, suggesting that our nebular line-width measurements trace the nuclear gravitational potential rather well. For values of $\sigma_{\mathrm{c}}$ between 90 and $220 \mathrm{~km} \mathrm{~s}^{-1}, 68 \%$ of our upper limits fall immediately above the $M_{\mathrm{BH}}-\sigma_{\mathrm{c}}$ relation without exceeding the expected $M_{\mathrm{BH}}$ values by more than a factor 4.1. No systematic trends or offsets are observed in this $\sigma_{\mathrm{c}}$ range as a function of the galaxy Hubble type or with respect to the presence of a bar. For 6 of our 12 upper limits on $M_{\mathrm{BH}}$ with $\sigma_{\mathrm{c}}<90 \mathrm{~km} \mathrm{~s}^{-1}$, our line-width measurements are more sensitive to the stellar contribution than to the gravitational potential, either due to the presence of a nuclear stellar cluster or because of a greater distance compared to the other galaxies at the low- $\sigma_{\mathrm{c}}$ end of the $M_{\mathrm{BH}}-\sigma_{\mathrm{c}}$ relation. Conversely, our $M_{\mathrm{BH}}$ upper bounds appear to lie closer to the expected $M_{\mathrm{BH}}$ in the most massive elliptical galaxies with values of $\sigma_{\mathrm{c}}$ above $220 \mathrm{~km} \mathrm{~s}^{-1}$. Such a flattening of the $M_{\mathrm{BH}}-\sigma_{\mathrm{c}}$ relation at its high- $\sigma_{\mathrm{c}}$ end would appear consistent with a coevolution of supermassive black holes and galaxies driven by dry mergers, although more consistent measurements for $\sigma_{\mathrm{c}}$ and $K$-band luminosity are needed for these kind of objects before systematic effects can be ruled out.

\section{Reference}

Beifiori, A., Sarzi, M., Corsini, E. M., Dalla Bontà, E., Pizzella, A., Coccato, L., \& Bertola, F. 2009, ApJ, 692, 856 